\title{
Many-point classical conformal blocks and geodesic networks on the hyperbolic plane
}

\author{
Konstantin Alkalaev \\ I.E. Tamm Department of Theoretical Physics, P.N. Lebedev Physical Institute, \\ Leninsky ave. 53, Moscow, 119991 Russia \\ Department of General and Applied Physics, Moscow Institute of Physics and Technology, \\ 7 Institutskiy per., Dolgoprudnyi, Moscow region, 141700 Russia \\ E-mail: alkalaev@lpi.ru
}

ABSTRACT: We study the semiclassical holographic correspondence between $2 d$ CFT $n$ point conformal blocks and massive particle configurations in the asymptotically $A d S_{3}$ space. On the boundary we use the heavy-light approximation in which case two of primary operators are the background for the other $(n-2)$ operators considered as fluctuations. In the bulk the particle dynamics can be reduced to the hyperbolic time slice. Although lacking exact solutions we nevertheless show that for any $n$ the classical $n$-point conformal block is equal to the length of the dual geodesic network connecting $n-3$ cubic vertices of worldline segments. To this end, both the bulk and boundary systems are reformulated as potential vector fields. Gradients of the conformal block and geodesic length are given respectively by accessory parameters of the monodromy problem and particle momenta of the on-shell worldline action represented as a function of insertion points. We show that the accessory parameters and particle momenta are constrained by two different algebraic equation systems which nevertheless have the same roots thereby guaranteeing the correspondence.

Keywords: AdS-CFT Correspondence, Conformal and W Symmetry, Conformal Field Theory, Classical Theories of Gravity

ARXIV EPRINT: 1610.06717 


\section{Contents}

1 Introduction 1

2 Classical blocks and the monodromy problem 3

3 Heavy-light perturbation theory $\quad 6$

$\begin{array}{lll}3.1 & \text { Solving the Fuchsian equation } & 7\end{array}$

3.2 Computing monodromies 8

$\begin{array}{lll}3.3 & \text { Eigenvalue condition } & 8\end{array}$

4 Geodesic networks on the hyperbolic plane $\quad 9$

$\begin{array}{lll}4.1 & \text { Cubic vertex and triangle inequalities } & 11\end{array}$

$\begin{array}{lll}4.2 & \text { Dual geodesic network } & 13\end{array}$

$\begin{array}{lll}4.3 & \text { Angular balance condition } & 14\end{array}$

5 Bulk/boundary correspondence $\quad 16$

$\begin{array}{ll}5.1 \text { Weak equivalence } & 17\end{array}$

$\begin{array}{lll}5.2 & \text { Accessory equations } & 18\end{array}$

$\begin{array}{lll}5.3 \text { Momentum equations } & 19\end{array}$

$\begin{array}{ll}5.4 \text { Comparing two systems } & 20\end{array}$

6 Conclusion $\quad 21$

A Technical details $\quad 22$

A.1 Worldlines in the angle deficit geometry 22

$\begin{array}{lll}\text { A.2 Proof of proposition } 4.1 & 23\end{array}$

A.3 Proof of proposition $5.3 \quad 24$

$\begin{array}{lll}\text { A.4 Proof of lemma } 5.4 & 25\end{array}$

A.5 Proof of lemma 5.5 25

\section{Introduction}

The AdS/CFT correspondence gives an effective prescription how to calculate CFT correlators from AdS action, at least in the saddle-point approximation [1-3]. It is interesting that the correspondence can be understood at a more structural level. CFT correlation functions can be decomposed into the theory-independent conformal blocks completely fixed by conformal symmetry. It is natural to question what are the bulk counterparts of conformal blocks. Recently, such dual objects were described in the case of $2 d$ CFT conformal blocks considered in the limit of infinite conformal parameters $\Delta, c \rightarrow \infty$ what corresponds to the semiclassical approximation on the gravity side. It was shown that on 
the Riemann sphere the limiting conformal blocks called classical are equally described as lengths of particular geodesic networks stretched in the asymptotically $A d S_{3}$ space [4-16]. The essential ingredient here is the heavy-light approximation, where two of primary operators form the background for the other operators [5]. Depending on their conformal dimensions the background operators produce the angle deficit or BTZ black hole in the bulk so that perturbative operators correspond to massive test particles.

The basic idea behind this kind of semiclassical AdS/CFT correspondence is quite simple. The semiclassical regime assumes that both the central charge and conformal dimensions tend to infinity such that ratios $\Delta / c$ are kept fixed. Then, the conformal block $F(z \mid \Delta, c)$ is exponentiated $F(z \mid \Delta, c) \sim \exp \left(\frac{1}{c} f\left(z \mid \frac{\Delta}{c}\right)\right)$ to yield the classical conformal block $f\left(z \mid \frac{\Delta}{c}\right)$ [17]. On the gravity side, the semiclassical path integral is dominated by classical paths describing geodesic motions of massive particles corresponding to primary operator insertions on the boundary [18-20].

The relation between classical conformal blocks and classical mechanics goes far beyond the AdS/CFT correspondence. Indeed, conformal blocks are known to be solutions of the Virasoro singular vector decoupling condition [21]. On the other hand, the conformal blocks with arbitrary conformal dimensions in the semiclassical limit were shown to satisfy the Painlevé VI equation which is just the decoupling condition represented in the HamiltonJacobi form [22]. It follows that constraining conformal dimensions within the heavy-light approximation the Painleve VI equation can be perturbatively reduced to the equation of motion for massive particles propagating in the asymptotically $A d S_{3}$ space.

The study of the singular vector decoupling condition brings to light many rich algebraic structures (see, e.g., [22, 23] and references therein). For example, the monodromy problem for the semiclassical decoupling condition expressed by the second order Fuchsian equation and the classical conformal blocks are deeply related [17, 21]. The Fuchsian equation $\psi^{\prime \prime}(z)+T(z) \psi(z)=0$ has the monodromy group fixed by the form of the stress-energy tensor $T(z)$, where conformal dimensions $\Delta$ are residues at the second order poles, while the accessory parameters $c_{i}$ are residues at the simple poles. On the other hand, the accessory parameters are gradients of the classical conformal block $c_{i}=\frac{\partial}{\partial z_{i}} f\left(z \mid \frac{\Delta}{c}\right)$. It follows that fixing the monodromy allows finding the classical conformal block.

Classical conformal blocks in the heavy-light approximation can be calculated within various approaches, e.g., using the monodromy method $[4,5,9,14,15]$, the Zamolodchikov recursion or the FKW global block method [9, 10, 24], or the AGT technique [12]. One way or another, the resulting block reproduces the length of dual geodesic network. However, it seems that only the monodromy method conceptually explains why the correspondence holds, see, e.g., the discussion in [9]. For example, the accessory parameter of the monodromy method can be interpreted as the momentum of a particle in the bulk whose worldline is attached to the conformal boundary. ${ }^{1}$ This is quite natural because the accessory parameter is the gradient of the classical conformal block, while external parti-

\footnotetext{
${ }^{1}$ The accessory parameters had previously emerged in connection with mechanical momenta. See, e.g., [25], where the hamiltonian structure of the $2+1$ gravity is discussed in the context of the so-called Polyakov conjecture and the Liouville theory.
} 
cle's momentum is the gradient of the on-shell worldline action. Exact relation based on the analysis of the accessory parameter/particle's momentum equations was given in [14].

Our analysis in this paper focuses on proving the AdS/CFT correspondence between classical conformal blocks and dual geodesic networks in the $n$-point case in the heavylight approximation. We show that many-point blocks and dual lengths coincide up to logarithmic terms related to the conformal map from the complex plane to cylinder. It is important to note that we do not find these functions explicitly. Instead, using the monodromy method on the boundary and the worldline approach in the bulk we prove that the two descriptions of $n$-point configurations are equivalent.

Intrinsically, the proof of the equivalence is reduced to considering a potential vector field $A_{i}(x)=\partial_{i} U(x)$, where $x_{i}$ are $n-2$ coordinates and $U(x)$ is a potential. Potential vector field systems underlie both the bulk and boundary analysis. On the boundary, using the monodromy method we identify the vector field components with the accessory parameters and the potential with the conformal block, while $x_{i}$ are insertion coordinates of $n-2$ perturbative primary operators. In the bulk, the network stretched in the angle deficit space has $n-2$ boundary attachment points $x_{i}$, the vector field components are particular components of canonical momenta of massive test particles, while the potential is the on-shell worldline action identified with the geodesic length.

Both the accessory parameters and momenta are subjected to complicated algebraic equations arising respectively as the monodromy conditions on the boundary and the least action principle in the bulk. Their exact solutions are known only in the 4-point case because the corresponding equation systems are reduced to just a quadratic equation [5, 9]. In many-point case only approximate solutions are available $[4,12,14,15]$. In this paper we prove that the two algebraic equation systems have the same roots. Therefore, the correspondence is guaranteed.

The paper is organized as follows. In section 2 we review the monodromy method for conformal blocks and fix our notation and conventions. In section 3 the monodromy problem is analyzed using the heavy-light perturbation theory. In section 4 we discuss the bulk space with the angle deficit and the dual geodesic network, see also appendix A.1. Here, we consider the Routh reduction of the bulk dynamics to the hyperbolic time slice, we study a vertex of three lines on the hyperbolic plane, and analyze the minimal length condition and the so-called angular balance condition. The bulk/boundary correspondence in the $n$-point case is shown in section 5. Proofs of lemmas and propositions formulated in sections 4.2 and 5 are collected in appendices A.2-A.5. In the concluding section 6 we discuss our main results and some future directions.

\section{Classical blocks and the monodromy problem}

There are two basic ideas to compute $n$-point classical conformal blocks using the monodromy method. First, conformal blocks in a given channel are eigenfunctions of the monodromy operator associated with a particular contour on the punctured region. Second, in the $c \rightarrow \infty$ limit a particular degenerate operator of the quantum $(n+1)$-point conformal block effectively decouples yielding the classical $n$-point conformal block. Comparing 


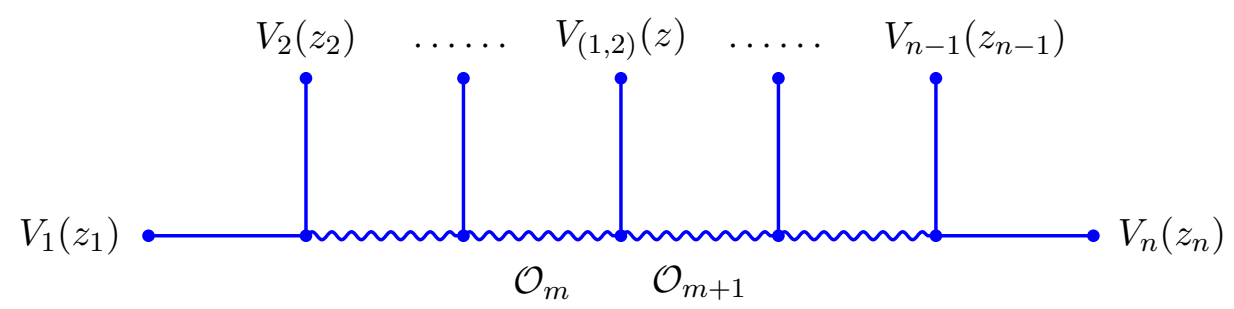

Figure 1. The $(n+1)$-point conformal block in a particular channel, where the degenerate operator fuses with $\mathcal{O}_{m}$ to yield $\mathcal{O}_{m+1}$ for any $m=1, \ldots, n-3$. There are $2(n-3)$ blocks of this type arising as solutions of the decoupling condition. In the limit $c \rightarrow \infty$ the degenerate operator decouples and therefore the only surviving block is given by that one shown on figure 2 . We fix $z_{1}=0$, $z_{n-1}=1, z_{n}=\infty$.

the monodromy matrices along particular contours computed before and after taking the semiclassical limit defines all gradients of the $n$-point classical conformal block in terms of coordinates of punctures. In this way, the problem is reduced to solving first order differential equations (for review, see, e.g., $[4,8,22]$ ).

We consider the $(n+1)$-point correlation function $\left\langle V_{(1,2)}(y) V_{1}\left(z_{1}\right) \cdots V_{n}\left(z_{n}\right)\right\rangle$ on the Riemann sphere with one second level degenerate operator of dimension $\Delta_{(1,2)}$ in point $y$ and $n$ general primaries of dimensions $\Delta_{i}$ in points $z_{i}, i=1, \ldots, n$. The correlation function satisfies the second order differential equation originating from the Virasoro singular vector decoupling condition [21]. The same is true for conformal blocks because the decoupling condition follows from Virasoro algebra only. The space of solutions is two-dimensional, hence the monodromy operators are $2 \times 2$ matrices. On the other hand, any $(n+1)$ point conformal block in a given channel has $n$ singular points and therefore there are $n$ independent monodromies.

We consider the OPE associated with the set of concentric contours around a common center $z_{1}=0$. Inserting the degenerate primary $V_{(1,2)}(y)$ between primaries $V_{k}\left(z_{k}\right)$ and $V_{k+1}\left(z_{k+1}\right)$ we fix a particular channel which means that $y$ should lie on the contour enclosing insertion points $z_{1}, \ldots, z_{m}$ :

$$
\text { Contour } \gamma_{k} \text { encircles points } \quad\left\{z_{1}, \ldots, z_{k+1}\right\}, \quad k=1, \ldots, n-3 \text {. }
$$

Then, points $z_{k+2}, \ldots, z_{n}$ are outside contour $\gamma_{k}$ and, therefore, $\gamma_{k} \subset \gamma_{k+1}$. The resulting channels are shown on figure 1.

Remarkably, the OPE ties monodromy of solutions around particular contours to dimensions of the exchanged operators in a particularly simple way. For the degenerate primary inserted as on figure 1 we find that the conformal block is dominated by $\left(z_{m}-y\right)^{\tilde{\Delta}_{m+1}-\Delta_{(1,2)}-\tilde{\Delta}_{m}}$. By the OPE argument, moving $y$ around $z_{m}$ is equivalent to moving around insertion points of those operators which have been fused into the exchanged operator. Thus, computing the monodromy of the above power-law function we easily find the monodromy along the contour $\gamma_{k}(2.1)$. 


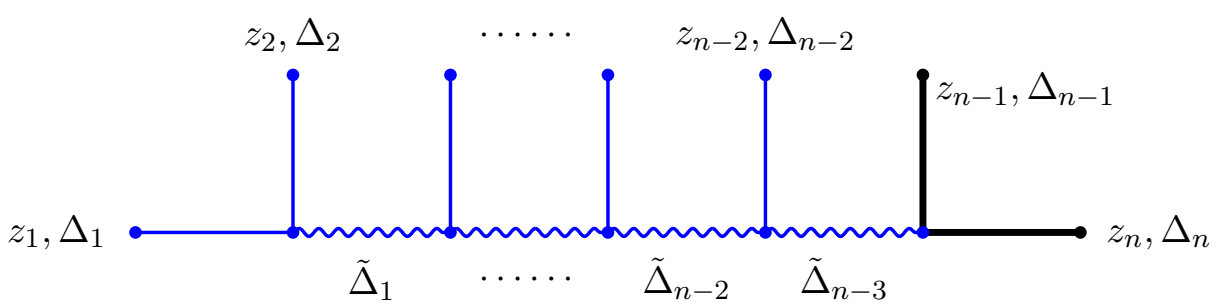

Figure 2. The $n$-point conformal block. Two bold black lines are background heavy operators, thin blue lines represent primary and exchanged perturbative heavy operators which are discussed in section 3 .

Indeed, using the Liouville parameterization ${ }^{2}$ we find that $\Delta_{(1,2)}=-1 / 2-3 b^{2} / 4$, while conformal dimensions of exchanged operators are related by the fusion rule as $\tilde{\Delta}_{m+1}-\tilde{\Delta}_{m}=$ $-b^{2} / 4 \pm i b P_{m}$ [21]. Then, the monodromy matrix associated with $\gamma_{k}$ is given by

$$
\widetilde{\mathbb{M}}\left(\gamma_{k}\right)=\left(\begin{array}{cc}
e^{2 \pi i M_{+k}} & 0 \\
0 & e^{2 \pi i M_{-k}}
\end{array}\right), \quad M_{ \pm k}=\frac{1}{2}+\frac{b^{2}}{2} \pm i b P_{k-1} .
$$

The classical conformal blocks arise in the limit when the central charge and conformal dimensions simultaneously tend to infinity. Both external and exchanged dimensions $\Delta_{m}$ and $\tilde{\Delta}_{n}$ grow linearly with the charge $c$ in such a way that ratios $\epsilon_{m}=6 \Delta_{m} / c$ and $\tilde{\epsilon}_{n}=$ $6 \tilde{\Delta}_{n} / c$ called classical dimensions remain fixed in $c \rightarrow \infty$. Then, the quantum conformal block is represented as an exponential of the classical conformal block [17]. Operators with fixed classical dimensions are heavy, while those with vanishing classical dimensions are light.

In our case of the $(n+1)$-point conformal block all operators are supposed to be heavy while the degenerate operator is $\operatorname{light} \lim _{b \rightarrow 0} \Delta_{(1,2)}=1 / 2$. Thus, in the semiclassical limit it decouples from the other operators, while adjacent exchanged dimensions get equal $\lim _{b \rightarrow 0}\left(\tilde{\Delta}_{m}-\tilde{\Delta}_{m+1}\right)=0$, see figure 1. The limiting $(n+1)$-point conformal block factorizes as

$$
\left.\mathcal{F}\left(y, z \mid \Delta_{m}, \tilde{\Delta}_{n}\right)\right|_{c \rightarrow \infty} \rightarrow \psi(y \mid z) \exp \left[-\frac{c}{6} f\left(z \mid \epsilon_{i}, \tilde{\epsilon}_{j}\right)\right],
$$

where we denoted $z=\left\{z_{1}, \ldots, z_{n}\right\}$, function $\psi(y \mid z)$ describes the semiclassical contribution of the degenerate operator, while the exponential factor $f\left(z \mid \epsilon_{i}, \tilde{\epsilon}_{j}\right)$ is the $n$-point classical conformal block which depends on external and exchanged classical conformal dimensions $\epsilon_{i}$ and $\tilde{\epsilon}_{j}[17]$ (see also $[4,8,17,22,26]$ ). We note that $(n+1)$-point conformal blocks can be considered in different channels arising from different ways to insert the degenerate operator between other operators, see figure 1. Such an ordering singles out a particular channel of the limiting $n$-point block (2.3), see figure 2 .

Function $\psi(y \mid z)$ satisfies the Fuchsian equation arising from the decoupling condition

$$
\left[\frac{d^{2}}{d y^{2}}+T(y \mid z)\right] \psi(y \mid z)=0, \quad \text { where } \quad T(y \mid z)=\sum_{i=1}^{n} \frac{\epsilon_{i}}{\left(y-z_{i}\right)^{2}}+\sum_{i=1}^{n} \frac{c_{i}}{y-z_{i}} .
$$

\footnotetext{
${ }^{2}$ We change $(\Delta, c) \rightarrow(P, b)$ according to $\Delta(P)=\frac{c-1}{24}+P^{2}$ and $c=1+6\left(b+b^{-1}\right)^{2}$ [21]. The limit $c \rightarrow \infty$ can equivalently be described as $b \rightarrow 0$.
} 
The accessory parameters $c_{i}$ are gradients of the classical $n$-point conformal block

$$
c_{i}(z)=\frac{\partial f(z)}{\partial z_{i}}, \quad i=1, \ldots, n,
$$

and satisfy the linear constraints

$$
\sum_{i=1}^{n} c_{i}=0, \quad \sum_{i=1}^{n}\left(c_{i} z_{i}+\epsilon_{i}\right)=0, \quad \sum_{i=1}^{n}\left(c_{i} z_{i}^{2}+2 \epsilon_{i} z_{i}\right)=0 .
$$

It follows that expanding the stress-energy tensor around $y=\infty$ we find no terms $1 / y^{l}$, $l=1,2,3$ and therefore near the infinity $T(y) \sim 1 / y^{4}$. Choosing $c_{2}, \ldots, c_{n-2}$ as independent parameters and fixing, accordingly, $z_{1}=0, z_{n-1}=1, z_{n}=\infty$, the above constraints are solved as $[14,15]$

$$
\begin{aligned}
c_{1} & =-\sum_{i=2}^{n-2}\left[c_{i}\left(1-z_{i}\right)-\epsilon_{i}\right]+\epsilon_{1}+\epsilon_{n-1}-\epsilon_{n}, \\
c_{n-1} & =-\sum_{i=2}^{n-2} c_{i} z_{i}+\epsilon_{n}-\sum_{i=1}^{n-1} \epsilon_{i}, \quad c_{n}=0 .
\end{aligned}
$$

Then, the stress-energy tensor $T(y \mid z)$ takes the form

$$
T(y \mid z)=\sum_{i=1}^{n-1} \frac{\epsilon_{i}}{\left(y-z_{i}\right)^{2}}+\sum_{i=2}^{n-2} c_{i} \frac{z_{i}\left(z_{i}-1\right)}{y\left(y-z_{i}\right)(y-1)}+\frac{\epsilon_{n}-\sum_{i=1}^{n-1} \epsilon_{i}}{y(y-1)} .
$$

The corresponding Fuchsian equation still has $n$ regular singular points around which we compute the monodromy. Continuing solutions $\psi(y \mid z)$ along the contours $\gamma_{k}(2.1)$ on the punctured $y$-plane and comparing the resulting monodromy matrices with (2.2) we can find the accessory parameters as functions of classical conformal dimensions and insertion points.

\section{Heavy-light perturbation theory}

Though the general solution to the Fuchsian equation with $n$ singular points is unknown we can try to use perturbation theory. The idea is to consider $k$ heavy operators as the background for the rest $n-k$ heavy operators. It follows that the corresponding dimensions are constrained as $\epsilon_{\text {pert }} / \epsilon_{\text {back }} \ll 1$, where $\epsilon_{\text {back }}$ are dimensions of the background operators, $\epsilon_{\text {pert }}$ are dimensions of the perturbative operators. Obviously, the simplest cases are given by $k=2$ or $k=3$ background operators. The case of $k=1$ background operator is trivial because one-point functions on the sphere are vanishing. In the $k=2$ case, the background conformal dimensions should be equal to each other for the corresponding two-point function to be non-vanishing. In order to apply perturbation theory in the $k \geq 4$ case we have to know exact solutions of the Fuchsian equation with $k$ singularities.

Following $[5,9,14,15]$ we consider the case of $k=2$ background operators (bold black lines on figure 2). Let $\epsilon_{n-1}=\epsilon_{n} \equiv \epsilon_{h}$ be the background heavy dimension, while $\epsilon_{i}$, 
$i=1, \ldots, n-2$ be perturbative heavy dimensions. It is assumed that $\epsilon_{i} / \epsilon_{h} \ll 1$. Then, the Fuchsian equation (2.4) with the stress-energy tensor (2.9) can be solved perturbatively. We expand all functions as

$$
\begin{aligned}
\psi(y, z) & =\psi^{(0)}(y, z)+\psi^{(1)}(y, z)+\psi^{(2)}(y, z)+\ldots, \\
T(y, z) & =T^{(0)}(y, z)+T^{(1)}(y, z)+T^{(2)}(y, z)+\ldots \\
c_{i}(z) & =c_{i}^{(0)}(z)+c_{i}^{(1)}(z)+c_{i}^{(2)}(z)+\ldots
\end{aligned}
$$

where expansion parameters are perturbative heavy dimensions. The accessory parameter expansion starts with terms linear in the conformal dimensions so that $c_{i}^{(0)}=0$. For the sake of simplicity, from now on we denote $c_{i}^{(1)}(z):=c_{i}(z)$. The classical conformal block (2.3) is similarly expanded,

$$
f(z)=f^{(0)}(z)+f^{(1)}(z)+f^{(2)}(z)+\ldots,
$$

in the way consistent with expansion (3.3) and relation (2.5). The zeroth approximation corresponds to the classical conformal block of 2-point functions of the background operators. Hence, $f^{(0)}(z)=0$ and the first non-trivial correction is given by $f^{(1)}(z)$. By analogy with the first order accessory parameters we denote $f^{(1)}(z):=f(z)$ and therefore the relation (2.5) remains unchanged.

\subsection{Solving the Fuchsian equation}

Using (3.1)-(3.3) we find that the perturbatively expanded Fuchsian equation yields a chain of inhomogeneous linear equations $\mathcal{D}_{0} \psi^{(s)}(y, z)+T^{(s)} \psi^{(s-1)}(y, z)=0, s=0,1,2, \ldots$, with a unique differential part given by the operator $\mathcal{D}_{0}=d^{2} / d y^{2}+T^{(0)}(y)$. The lowest order equations are given by

$$
\mathcal{D}_{0} \psi^{(0)}(y, z)=0, \quad \mathcal{D}_{0} \psi^{(1)}(y, z)+T^{(1)} \psi^{(0)}(y, z)=0,
$$

where the stress-energy components are read off from (2.9)

$$
T^{(0)}(y)=\frac{\epsilon_{h}}{(y-1)^{2}}, \quad T^{(1)}(y, z)=\sum_{i=1}^{n-2} \frac{\epsilon_{i}}{\left(y-z_{i}\right)^{2}}+\sum_{i=2}^{n-2} c_{i} \frac{z_{i}\left(z_{i}-1\right)}{y\left(y-z_{i}\right)(y-1)}-\frac{\sum_{i=1}^{n-2} \epsilon_{i}}{y(y-1)},
$$

with the convention $z_{1}=0$. There are two zeroth order branches $\psi_{ \pm}^{(0)}(y, z)=(1-y)^{(1 \pm \alpha) / 2}$, where

$$
\alpha=\sqrt{1-4 \epsilon_{h}} .
$$

Using the zeroth order solution we find in the first order that

$$
\psi_{ \pm}^{(1)}(y, z)=\frac{1}{\alpha} \psi_{+}^{(0)}(y) \int d y \psi_{-}^{(0)}(y) T^{(1)}(y, z) \psi_{ \pm}^{(0)}(y)-\frac{1}{\alpha} \psi_{-}^{(0)}(y) \int d y \psi_{+}^{(0)}(z) T^{(1)}(y, z) \psi_{ \pm}^{(0)}(y) .
$$

Corrections $\psi_{ \pm}^{(1)}$ have branch points inherited from those of $\psi_{ \pm}^{(0)}(y)$ and $T(y, z)$. 


\subsection{Computing monodromies}

Now, we continue the perturbative solution $\psi=\psi^{(0)}+\psi^{(1)}+\ldots$ along particular contours (2.1). Encircling the branch points of the solution we define the monodromy as $\mathbb{M}(\gamma): \psi \rightarrow \mathbb{M}(\gamma) \psi$, where the monodromy matrix is also expanded as $\mathbb{M}=\mathbb{M}_{0}+\mathbb{M}_{1}+\ldots$ In components,

$$
\gamma_{k}:\left(\begin{array}{c}
\psi_{+} \\
\psi_{-}
\end{array}\right) \rightarrow\left(\begin{array}{ll}
M_{++}\left(\gamma_{k}\right) & M_{+-}\left(\gamma_{k}\right) \\
M_{-+}\left(\gamma_{k}\right) & M_{--}\left(\gamma_{k}\right)
\end{array}\right)\left(\begin{array}{c}
\psi_{+} \\
\psi_{-}
\end{array}\right) .
$$

Expanding both the solution and the monodromy matrix as above we find that the zeroth order matrix $\mathbb{M}_{0}$ defines the monodromy of $\psi^{(0)}(y)$ with the branch point $y=1$. However, the contours $\gamma_{k}$ (2.1) do not enclose this point, and, therefore, $\mathbb{M}_{0}=\mathbb{I}$. It follows that the perturbative solution represented as a linear combination of the zeroth order solutions with the integral coefficients (3.8) fits well the monodromy computation. Indeed, the whole computation is reduced to evaluating the integrals along the contours $\gamma_{k}$,

$$
\begin{aligned}
& I_{ \pm+}^{(k)}(z)=+\frac{1}{\alpha} \int_{\gamma_{k}} d y \psi_{-}^{(0)}(y) T^{(1)}(y, z) \psi_{ \pm}^{(0)}(y) \\
& I_{ \pm-}^{(k)}(z)=-\frac{1}{\alpha} \int_{\gamma_{k}} d y \psi_{ \pm}^{(0)}(y) T^{(1)}(y, z) \psi_{-}^{(0)}(y)
\end{aligned}
$$

Substituting the stress-energy tensor correction $T^{(1)}(y, z)$ given by (3.6) and using the residue theorem we have

$$
\begin{aligned}
& I_{+-}^{(k)}=\frac{2 \pi i}{\alpha}\left[\alpha \epsilon_{1}+\sum_{i=2}^{n-2}\left(c_{i}\left(1-z_{i}\right)-\epsilon_{i}\right)-\sum_{i=2}^{k+1}\left(1-z_{i}\right)^{\alpha}\left(c_{i}\left(1-z_{i}\right)-\epsilon_{i}(1+\alpha)\right)\right], \\
& I_{++}^{(k)}=\frac{2 \pi i}{\alpha} \sum_{i=k+2}^{n-2}\left[c_{i}\left(1-z_{i}\right)-\epsilon_{i}\right], \quad I_{-+}^{(k)}=\left.I_{+-}^{(k)}\right|_{\alpha \rightarrow-\alpha}, \quad I_{--}^{(k)}=\left.I_{++}^{(k)}\right|_{\alpha \rightarrow-\alpha} .
\end{aligned}
$$

For a given number of insertion points $n$ integrals $I_{++}$and $I_{--}$over the maximal contour $\gamma_{n-3}$ are always zero, $I_{++}^{(n-3)}=-I_{--}^{(n-3)}=0$. It follows that elements of the first order correction $\mathbb{M}_{1}$ are just the contour integrals, $M_{ \pm \pm}\left(\gamma_{k}\right)=I_{ \pm \pm}^{(k)}$. The second order monodromy matrix $\mathbb{M}=\mathbb{M}_{0}+\mathbb{M}_{1}$ is therefore given by

$$
\mathbb{M}\left(\gamma_{k}\right)=\left(\begin{array}{cc}
1+I_{++}^{(k)} & I_{+-}^{(k)} \\
I_{-+}^{(k)} & 1+I_{--}^{(k)}
\end{array}\right) .
$$

\subsection{Eigenvalue condition}

Matrices $\mathbb{M}\left(\gamma_{k}\right)$ (3.14) on the one hand and matrices $\widetilde{\mathbb{M}}\left(\gamma_{k}\right)(2.2)$ at infinite central charge and small perturbative classical dimensions on the other hand describe the same monodromy associated to continuation of the degenerate operator along the contours $\gamma_{k}$. Equating the corresponding eigenvalues we arrive at the system of equations on conformal dimensions of exchanged operators, insertion points, and accessory parameters. 
We consider the monodromy (2.2) semiclassically. The limiting matrix reads

$$
\lim _{b \rightarrow 0} \widetilde{\mathbb{M}}\left(\gamma_{k}\right)=\left(\begin{array}{cc}
e^{2 \pi i M_{-k}} & 0 \\
0 & e^{2 \pi i M_{+k}}
\end{array}\right), \quad M_{ \pm k}=\frac{1}{2} \pm \frac{1}{2} \sqrt{1-4 \tilde{\epsilon}_{k}} .
$$

Within the perturbation theory, the eigenvalues of (3.15) up to linear order in dimensions of the exchanged operators are given by $\lambda_{ \pm k}=1 \pm 2 \pi i \tilde{\epsilon}_{k}$. To diagonalize matrices (3.14) we solve the characteristic equation $\operatorname{det}\left(\mathbb{M}\left(\gamma_{k}\right)-\lambda_{k} \mathbb{I}\right)=0$. Using (3.13) we find that the eigenvalues are defined by the quadratic equation $\left(1-\lambda_{k}\right)^{2}=I_{++}^{(k)}+I_{+-}^{(k)} I_{-+}^{(k)}$. Equating two sets of eigenvalues we arrive at the following system

$$
\left(I_{++}^{(k)}\right)^{2}+I_{-+}^{(k)} I_{+-}^{(k)}=-4 \pi^{2} \tilde{\epsilon}_{k}^{2}, \quad k=1, \ldots, n-3 .
$$

In what follows, equations (3.16) supplemented by equations (2.7) are referred to as the accessory equations. Recalling the form of the contour integrals (3.12)-(3.13) we find out that (3.16) is the system of quadratic equations with coefficients depending on punctures $z_{k}$ and conformal dimensions $\alpha, \epsilon_{i}, \tilde{\epsilon}_{j}$. Solving them we can unambiguously express the accessory parameters as functions of conformal dimensions and coordinates of the punctures. We discuss the accessory parameters equations in section 5.2 and their dual interpretation in section 5. Here we stress that classical conformal dimensions of external and exchanged operators in (3.16) are arbitrary. However, we can impose various constraints to facilitate solving the accessory parameters equations using approximation techniques [5, 9, 14, 15, 24].

\section{Geodesic networks on the hyperbolic plane}

Perturbative conformal blocks can be represented in terms of massive point particles propagating in the three-dimensional space described by the metric [5]

$$
d s^{2}=\frac{\alpha^{2}}{\cos ^{2} \rho}\left(d t^{2}+\sin ^{2} \rho d \phi^{2}+\frac{1}{\alpha^{2}} d \rho^{2}\right)
$$

where $t \in \mathbb{R}, \rho \in[0, \pi / 2], \phi \in[0,2 \pi)$. The space contains a conical singularity measured by the angle deficit $2 \pi(1-\alpha)$, where $\alpha \in(0,1]$. The metric (4.1) describes the constant negative curvature space with the topology $\mathbb{R} \times \mathcal{C}_{2}$. Two-dimensional slices $\phi=$ const. and $t=$ const. are also negative curvature spaces identified with the punctured hyperbolic plane, where the puncture corresponds to the conical singularity projected on two dimensions. The conformal boundary reached at $\rho \rightarrow \pi / 2$ is the Euclidean cylinder with local coordinates $t$ and $\phi$. The presence of conical singularity breaks the global $A d S_{3}$ isometry $(\alpha=1)$ down to Abelian isometry $\mathbb{R} \oplus o(2)$ generated by two Killing vectors $\partial_{t}$ and $\partial_{\phi}$. On the conformal boundary, the Abelian isometry is enhanced to full Virasoro algebra.

A massive particle on the angle deficit space with the interval (4.1) is described by the worldline action $S=\epsilon \int d \lambda \sqrt{g_{t t} \dot{t}^{2}+g_{\phi \phi} \dot{\phi}^{2}+g_{\rho \rho} \dot{\rho}^{2}}$, where $\epsilon$ is a classical conformal dimension identified with a mass, the metric coefficients are read off from (4.1), $\lambda$ is the evolution parameter and the dot denotes differentiation with respect to $\lambda$, see appendix A.1 for more details. The Abelian isometry guarantees that coordinates $\phi$ and $t$ are cyclic, i.e. 


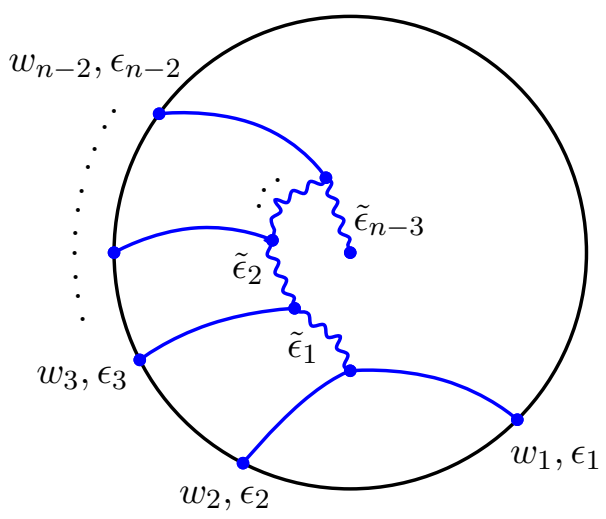

Figure 3. Network of geodesic lines on the hyperbolic disk. Solid and wave lines denote respectively external $\left(\epsilon_{m}\right)$ and exchanged $\left(\tilde{\epsilon}_{k}\right)$ particles, dotted lines denote the middle part of the graph. The boundary attachment points are $w_{m}, m=1, \ldots, n-2$.

$\delta S / \delta \phi \equiv 0$ and $\delta S / \delta t \equiv 0$. It follows that the original mechanics can be reduced to a simpler system described by the Routhian function, which means that we have to perform a partial Legendre transformation with respect to $\dot{\phi}$ and $\dot{t}$. Choosing the partial constraint $\dot{t}=0$ we arrive at the Routhian action, which describes a massive particle moving on the punctured hyperbolic disk,

$$
S=\int d \lambda L, \quad L=\epsilon \sqrt{\alpha^{2} \tan ^{2} \rho \dot{\phi}^{2}+\sec ^{2} \rho \dot{\rho}^{2}} .
$$

The residual isometry is given by $\operatorname{sl}(2, \mathbb{R})$ at $\alpha=1$ and $o(2)$ at $\alpha \neq 1$.

We consider a set of massive point particles propagating around the conical singularity. They interact to each other forming cubic vertices of worldlines. Of course, there are other possible types of interaction including quartic and higher vertices. However, the block/length correspondence singles out only cubic vertices. There are $2 n-5$ particles corresponding to the total number of external/exchanged lines of the dual $n$-point conformal block diagram shown on figure 2. External $n-2$ worldlines are attached to the conformal boundary at fixed points $w=\left(w_{1}, \ldots, w_{n-2}\right)$, where $w=\phi+i t$. Exchanged $n-3$ worldlines are stretched between vertices except for the radial line which ends at the center of the disk. The resulting geodesic network on the hyperbolic disk is shown on figure 3 [12]. It can be obtained by copy-pasting the conformal block diagram on figure 2 into the disk so that two background operator lines shrink to the point identified with the center of the disk, while the insertion points of conformal primaries go to the boundary attachment points. $^{3}$

Geodesics on the hyperbolic time slice (4.2) are most easily described using the Poincare disk model. In this case, these are segments of circles perpendicular to the boundary, including circles of infinite radius (the radial line on figure 3). Geodesic lengths are explicitly known as functions of endpoints, see, e.g., formula (A.4).

\footnotetext{
${ }^{3}$ The geodesic network on figure 3 can be obtained through the geodesic Witten diagram studied in $[13,27]$.
} 


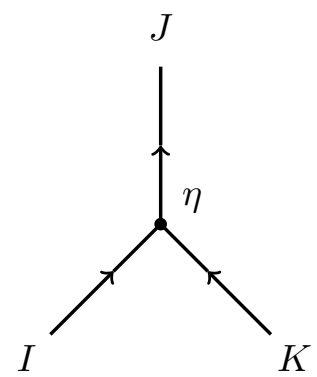

Figure 4. Cubic vertex on the hyperbolic disk. Incoming $(I, K)$ and outcoming $(J)$ momenta are constrained by the equilibrium condition. The radial vertex position is parameterized by $\eta=\cot ^{2} \rho$, where $\rho$ is the radial distance from the center.

\subsection{Cubic vertex and triangle inequalities}

Any vertex of the geodesic network on figure 3 connects three external and/or exchanged lines, where the vertex point is locally given by three coinciding inner endpoints while outer endpoints are free, see figure 4 . The vertex action for three distinct lines has the form

$$
S_{\star}=\epsilon_{I} \int_{O_{I}}^{\bullet} d \lambda L_{I}+\epsilon_{J} \int_{{ }_{O} J}^{\bullet} d \lambda L_{J}+\epsilon_{K} \int_{\mathrm{O}_{K}}^{\bullet} d \lambda L_{K}, \quad I \neq J \neq K,
$$

where each term is the worldline action on the hyperbolic disk (4.2) with the vertex point - and outer endpoints $o_{A}$, where $A=I, J, K$. The least principle guarantees that the geodesic segments satisfy the equilibrium condition at the vertex point

$$
P^{(I)}+P^{(J)}+P^{(K)}=0,
$$

where $P_{m}^{(A)}=\partial L_{A} / \partial \dot{X}_{(A)}^{m}$ are canonical momenta of three particles with coordinates $X_{(A)}^{m}$, where $m=\rho, \phi$ and $A=I, J, K$.

The equilibrium condition is conveniently parameterized by classical conformal dimensions $\epsilon_{A}$ and angular parameters

$$
s_{A}=\frac{\left|P_{\phi}^{(A)}\right|}{\alpha} .
$$

The parameter $s_{A}$ is an integration constant characterizing the form of a particular segment (see appendix A.1). Since $P_{\phi}^{(A)}= \pm \alpha s_{A}$, where the overall sign depends on the direction of the flow, we find that the radial and angular projections of (4.4) are given by

$$
\begin{array}{r}
\epsilon_{I} \sqrt{1-s_{I}^{2} \eta}-\epsilon_{J} \sqrt{1-s_{J}^{2} \eta}+\epsilon_{K} \sqrt{1-s_{K}^{2} \eta}=0, \\
\epsilon_{I} s_{I}+\epsilon_{J} s_{J}-\epsilon_{K} s_{K}=0
\end{array}
$$

where we expressed radial velocities through the vertex position according to (A.3).

Using the linear relation (4.7) we solve the radical equation (4.6) as follows

$$
\eta=\frac{1-\sigma_{I J}^{2}}{s_{I}^{2}+s_{J}^{2}-2 \sigma_{I J} s_{I} s_{J}}, \quad \text { where } \quad \sigma_{I J}=\frac{\epsilon_{I}^{2}+\epsilon_{J}^{2}-\epsilon_{K}^{2}}{2 \epsilon_{I} \epsilon_{J}} .
$$


Remarkably, there are no other roots. Indeed, the radical equation (4.6) can be solved by isolating one of radicals on one side and then squaring both sides. The resulting equation is linear in $\eta$. For example, the representation (4.8) is obtained by isolating the first radical in (4.6). Other equivalent forms of the vertex position can be obtained by isolating the second and third radicals,

$$
\begin{array}{ll}
\eta=\frac{1-\sigma_{I K}^{2}}{s_{I}^{2}+s_{K}^{2}-2 \sigma_{I K} s_{I} s_{K}}, & \text { where } \quad \sigma_{I K}=\frac{\epsilon_{I}^{2}+\epsilon_{K}^{2}-\epsilon_{J}^{2}}{2 \epsilon_{I} \epsilon_{K}}, \\
\eta=\frac{1-\sigma_{J K}^{2}}{s_{J}^{2}+s_{K}^{2}+2 \sigma_{J K} s_{J} s_{K}}, & \text { where } \quad \sigma_{J K}=\frac{\epsilon_{J}^{2}+\epsilon_{K}^{2}-\epsilon_{I}^{2}}{2 \epsilon_{J} \epsilon_{K}} .
\end{array}
$$

Note that equations (4.6) and (4.7) are linear combinations of the same type terms, but with different signs. This is why $\sigma_{J K}$ in (4.10) has a different sign.

The radicals in (4.6) impose restrictions on the radial vertex position

$$
0 \leq \eta \leq 1 / s_{A}^{2}, \quad A=I, J, K .
$$

The region $\eta<0$ is unphysical corresponding to imaginary values of the radial position. Examining the region $\eta \geq 0$ we find out that the classical conformal dimensions necessarily satisfy the triangle inequalities.

Proposition 4.1 The reality condition $\eta \geq 0$ is satisfied iff

$$
\begin{gathered}
\epsilon_{I}+\epsilon_{J} \geq \epsilon_{K}, \\
\epsilon_{I}+\epsilon_{K} \geq \epsilon_{J}, \\
\epsilon_{J}+\epsilon_{K} \geq \epsilon_{I} .
\end{gathered}
$$

The proof is given in appendix A.2. ${ }^{4}$ From the triangle inequalities (4.12) it follows that the sigmas (4.8)-(4.10) can be parameterized as cosines $\sigma_{A B}=\cos \gamma_{A B}$, where $\gamma_{A B}$ is the angle between $\epsilon_{A}$ and $\epsilon_{B}$ sides of the triangle (4.12) in the space of conformal dimensions. Introducing $s_{A B}^{2}=s_{A}^{2}+s_{B}^{2} \pm 2 \sigma_{A B} s_{A} s_{B}$ according to (A.9) we represent the vertex position (4.8)-(4.10) as

$$
\eta=\left[\frac{\sin \gamma_{I J}}{s_{I J}}\right]^{2}=\left[\frac{\sin \gamma_{I K}}{s_{I K}}\right]^{2}=\left[\frac{\sin \gamma_{J K}}{s_{J K}}\right]^{2} .
$$

In this form, it is similar to the law of sines in planar trigonometry. Nevertheless, unlike the conformal dimensions, the angular parameters $s_{I}, s_{J}, s_{K}$ do not form a triangle because, generally, $s_{A B} \neq s_{C}$. It would be interesting to understand the role of the ratio $(\sin \gamma) / s$ as an invariant of the geometry of cubic vertices on the hyperbolic space. Here we just note that the triangle inequalities (4.12) are analogous to triangle inequalities satisfied by conformal dimensions of primary operators in the semiclassical limit of the DOZZ threepoint correlation function [28]. Note that the triangle inequalities in the Liouville theory are supplemented by the Seiberg bound [29] and the Gauss-Bonnet constraint [28]. All together they guarantee that the Liouville field solution is real.

\footnotetext{
${ }^{4}$ The triangle inequalities (4.12) are also satisfied by the vertex of two background heavy operators of dimension $\epsilon_{h} \equiv \epsilon_{n-1}=\epsilon_{n}$ and the perturbative heavy exchanged operator of dimension $\tilde{\epsilon}_{n-3}$. In this case, $2 \epsilon_{h} \gg \tilde{\epsilon}_{n-3}$ and $\tilde{\epsilon}_{n-3} \geq 0$.
} 


\subsection{Dual geodesic network}

Gluing together $n-3$ vertices $\eta_{1}, \ldots, \eta_{n-3}$ with endpoints attached to $n-2$ boundary points $w=\left(w_{1}, \ldots, w_{n-2}\right)$ and the center of the disk we obtain the network shown on figure 3. At this stage all segments are naturally divided into external and exchanged ones. In what follows we use the condensed index $A=1, \ldots, 2 n-5$ :

$$
\{A\}=\{i, \tilde{j}\}: \quad i=1, \ldots, n-2, \quad \tilde{j}=1, \ldots, n-3,
$$

to label $n-2$ external and $n-3$ exchanged segments. The total worldline action is given by the sum of $n-3$ vertex actions (4.3), i.e., $S=\sum_{m=1}^{n-3} S_{\star}^{(m)}$, where endpoints are connected to each other in such a way to form the network shown on figure 3 . The resulting action reads

$$
S=\sum_{A=1}^{2 n-5} \epsilon_{A} \int_{\circ A}^{\bullet A} d \lambda L_{A},
$$

where Lagrangian $L_{A}$ describes $A$-th geodesic external/exchanged segment with endpoints ${ }_{O} A$ and $\bullet A$ defined by the form of the network. Any vertex $\eta_{i}, i=1, \ldots, n-3$ joins lines with labels $I=i+1, J=\tilde{i}, K=\widetilde{i-1}$, where, for convenience, we equated $\tilde{0}=1$.

Given that the action functional $S$ is stationary we find the equilibrium conditions (4.4) at each vertex point

$$
P_{(i+1)}+P_{(\tilde{i})}+P_{\widetilde{(i-1)}}^{\widetilde{2}}=0, \quad i=1, \ldots, n-3,
$$

and out-flowing momenta in all attachment points on the boundary and at the center of the disk,

$$
P_{(A)}=\frac{\partial S}{\partial X_{(A)}}, \quad A=1, \ldots, n-3, \widetilde{n-3},
$$

where the last equality is assumed to be weak, i.e. the action $S$ is evaluated on-shell.

All the attachment points have limiting radial positions, $\rho=\pi / 2$ for the boundary points and $\rho=0$ for the center of the disk. It follows that radial components of (4.17) trivialize because the corresponding variation terms do not contribute to the variation $\delta S$. The on-shell action of the network depends on angles of the boundary attachment points and the center of the disk, $S=S\left(w, \phi_{o}\right)$. Using (4.5) we can represent non-vanishing components of (4.17) as angular gradients of the on-shell action

$$
s_{i}=\frac{1}{\alpha \epsilon_{i}} \frac{\partial S}{\partial w_{i}}, \quad i=1, \ldots, n-2,
$$

and

$$
\tilde{s}_{n-3}=\frac{1}{\alpha \epsilon_{i}} \frac{\partial S}{\partial \phi_{o}} .
$$

The angular coordinate $\phi_{o}$ of the center is arbitrary, then the derivative in (4.19) vanishes, and, therefore,

$$
\tilde{s}_{n-3}=0
$$


It follows that the corresponding worldline is radial. Recalling that the center contains the conical singularity we conclude that the radial fall of the outer exchanged particle is quite natural.

Angular components of the equilibrium conditions (4.16) can be explicitly written as

$$
\epsilon_{i} s_{i}+\tilde{\epsilon}_{i-1} \tilde{s}_{i-1}-\tilde{\epsilon}_{i-2} \tilde{s}_{i-2}=0, \quad i=2, \ldots, n-1 .
$$

We can then solve (4.21) to obtain

$$
\tilde{\epsilon}_{k} \tilde{s}_{k}=\epsilon_{1} s_{1}-\sum_{i=2}^{k+1} \epsilon_{i} s_{i}, \quad k=1, \ldots, n-3,
$$

and therefore all exchanged momenta can be expressed in terms of the external ones. Taking $k=n-3$ we find out that the total outflowing angular parameter is zero. Indeed, in this case the right-hans side of (4.22) is the sum of outflowing parameters at the boundary attachment points, while the left-hand side is the outflowing parameter at the center of the disk (4.20). Thus, all external parameters are linearly related as

$$
-\epsilon_{1} s_{1}+\sum_{i=2}^{n-2} \epsilon_{i} s_{i}=0
$$

Choosing again $I=\widetilde{k-2}, J=\widetilde{k-1}, K=k$ we find that radial components of the equilibrium conditions (4.16) can be explicitly written as

$$
\tilde{\epsilon}_{k-1} \sqrt{1-\tilde{s}_{k-1}^{2} \eta_{k-1}}-\tilde{\epsilon}_{k-2} \sqrt{1-\tilde{s}_{k-2}^{2} \eta_{k-1}}+\epsilon_{k} \sqrt{1-\tilde{s}_{k}^{2} \eta_{k-1}}=0
$$

Vertex positions $\eta_{k-1}$ are directly read off from the general formula (4.8), namely

$$
\eta_{k-1}=\frac{1-\sigma_{k}^{2}}{s_{k}^{2}+\tilde{s}_{k-2}^{2}-2 \sigma_{k} s_{k} \tilde{s}_{k-2}}, \quad \sigma_{k}=\frac{\epsilon_{k}^{2}+\tilde{\epsilon}_{k-2}^{2}-\tilde{\epsilon}_{k-1}^{2}}{2 \epsilon_{k} \tilde{\epsilon}_{k-2}}, \quad k=2, \ldots, n-2 .
$$

Equivalent representations of $\eta_{k-1}$ can be obtained using (4.9) and (4.10).

\subsection{Angular balance condition}

We study angular positions of the endpoints which define the limits of integration in the worldline action (4.15). Let $\psi_{i}$ be angles of vertices $\eta_{i}, i=1, \ldots, n-3$ and $w_{i}, i=$ $1, \ldots, n-2$ be angles of the boundary attachment points, see figure 5. Generally, exchanged lines are stretched between two neighboring vertices while external lines connect vertices with boundary points. From figure 5 we find that the angular separation of the $i$-th external segment is

$$
\Delta \phi_{i}=w_{i}-\psi_{i-1}, \quad i=2, \ldots, n-2,
$$

while the angular separation of the $i$-th exchanged segment is

$$
\Delta \tilde{\phi}_{i}=\psi_{i+1}-\psi_{i}, \quad i=1, \ldots, n-4
$$




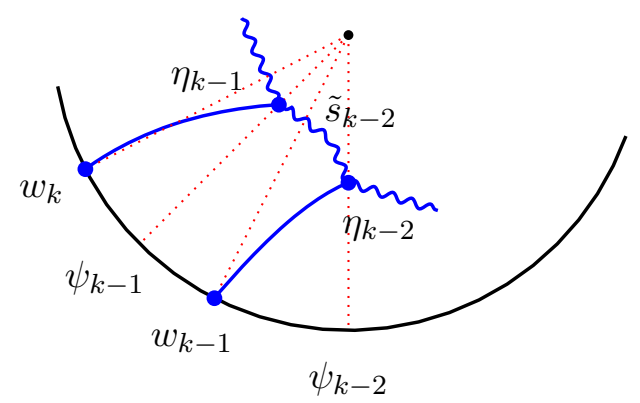

Figure 5. Angular separations. Dotted lines show angular positions $w_{i}$ and $\psi_{i}$ of the boundary attachment points and vertices, respectively.

Both the rightmost and leftmost parts of the network on figure 3 are different from the general pattern on figure 5 . Therefore, we identify $w_{1}=\psi_{0}$ and hence $\Delta \tilde{\phi}_{0}=\psi_{1}-\psi_{0}$ is the angular separation of the first external line. Also, $\Delta \tilde{\phi}_{n-3}=0$ because the outer exchanged line is radial. From figure 5 we find that angular positions satisfy the balance equation

$$
\left(w_{k}-w_{k-1}\right)+\Delta \phi_{k-1}=\Delta \phi_{k}+\Delta \tilde{\phi}_{k-2}, \quad k=2, \ldots, n-2 .
$$

Angular separation of the geodesic segment with two endpoints having radial and angular positions $\left(\phi^{\prime}, \eta^{\prime}\right)$ and $\left(\phi^{\prime \prime}, \eta^{\prime \prime}\right)$ and characterized by the angular parameter $s$ can be represented as [12]

$$
i \alpha\left(\phi^{\prime \prime}-\phi^{\prime}\right)=\ln \frac{\sqrt{1-s^{2} \eta^{\prime \prime}}-i s \sqrt{1+\eta^{\prime \prime}}}{\sqrt{1-s^{2} \eta^{\prime}}-i s \sqrt{1+\eta^{\prime}}}
$$

cf. (A.5). Boundary attachment points have $\eta=0$, radial vertex positions $\eta_{k}$ are given by (4.25). From the technical perspective, the logarithmic representation (4.29) is well suitable for the bulk/boundary correspondence analysis of section 5 because the conformal map from the complex plane to the boundary cylinder is also logarithmic (5.1).

Substituting (4.29) into the angular balance equation (4.28) we obtain the system of radical equations

$$
e^{i \alpha\left(w_{k}-w_{k-1}\right)} \frac{1-i s_{k}}{1-i s_{k-1}} \frac{D_{k-1}^{-}}{D_{k}^{+}}=1, \quad k=2, \ldots, n-2
$$

where we introduced notation

$$
\begin{aligned}
& D_{k}^{-}=\left(\sqrt{1-s_{k}^{2} \eta_{k-1}}-i s_{k} \sqrt{1+\eta_{k-1}}\right)\left(\sqrt{1-\tilde{s}_{k-1}^{2} \eta_{k-1}}-i \tilde{s}_{k-1} \sqrt{1+\eta_{k-1}}\right), \quad k=1, \ldots, n-2, \\
& D_{k}^{+}=\left(\sqrt{1-s_{k}^{2} \eta_{k-1}}-i s_{k} \sqrt{1+\eta_{k-1}}\right)\left(\sqrt{1-\tilde{s}_{k-2}^{2} \eta_{k-1}}-i \tilde{s}_{k-2} \sqrt{1+\eta_{k-1}}\right), \quad k=2, \ldots, n-2 .
\end{aligned}
$$


The left-hand side of (4.30) is unimodular so that the corresponding real and imaginary parts are not independent. Thus, the complex equations (4.30) are equivalent to original real equations (4.28). ${ }^{5}$

The angular balance equation (4.30) is too complicated because of radicals depending on vertex radial positions, which in turn depend non-trivially on angular paprameters (4.25). The roots of the system are yet unknown except for the simplest $n=4$ case $[5,9]$, where the solution can be found exactly, and the $n=5$ case $[12,14]$, where only perturbative solutions are available. ${ }^{6}$ In the next section we show that the dual network equations (4.21), (4.25), (4.30) have roots coinciding with those of the accessory parameter equations (2.7), (3.16).

\section{Bulk/boundary correspondence}

The correspondence is most clear in the case of 2-point correlation function of heavy background operators. Two operators inserted in $z=1$ and $z=\infty$ produce an asymptotically $A d S_{3}$ geometry (4.1) in the bulk with cylindrical conformal boundary. Angle deficit $\alpha$ in the metric (4.1) is related to the background operator conformal dimension by (3.7). This can be explicitly seen using the three-dimensional metric of asymptotically $A d S_{3}$ spacetime in the Banados form [30], where the stress-energy tensor is taken to be the background value $T^{(0)}(z)(3.6)$ (see $[6,10]$ for more details). Note that the CFT we started with is originally defined on the sphere. However, puncturing the sphere twice we obtain the once punctured complex plane, which can be conformally mapped on the cylinder. Opposite ends of the resulting infinitely long cylinder correspond to the background operator insertions. ${ }^{7}$

Let $(z, \bar{z})$ be coordinates on the punctured complex plane and $(w, \bar{w})$ be coordinates on the boundary cylinder. Then, the conformal map is given by

$$
w=i \ln (1-z) .
$$

In our case, the boundary attachment points $w_{i}$ are located on a circle obtained by slicing the boundary cylinder, see figure 3 . It follows that insertion points $z_{i}$ belong to the unit circle

$$
\left(z_{i}-1\right)\left(\bar{z}_{i}-1\right)=1, \quad i=1, \ldots, n-2 .
$$

In particular, $z_{1}=0$ fixed by projective $s l(2, \mathbb{C})$ isometry of the plane goes to $w_{1}=0$ fixed by $o(2)$ isometry transformation of the boundary circle.

We argue that the correspondence can be shown without knowing explicit expressions for conformal blocks/geodesic lengths. We have seen that both classical the conformal block and the geodesic length are defined through auxiliary parameters subjected to algebraic equation systems. It is natural to question whether two equation systems are equivalent provided that the conformal block and geodesic length are related by the conformal map.

\footnotetext{
${ }^{5}$ Exceedingly lengthy but equivalent representation of the angular equations was also given in [12].

${ }^{6}$ Approximate solutions in other conformal block channels were also considered in [5, 15].

${ }^{7}$ This topological consideration can be rigorously implemented within the Liouville theory with heavy insertions, see, e.g., [31].
} 
We might hope that they are literally the same. However, it turns out that the equivalence is weaker. It is claimed only that being generally different the two systems have the same roots. This section addresses such an equivalence in the $n$-point case.

The correspondence between a CFT with two background operators in the infinite central charge limit and dual geodesic networks on the angle deficit background claims that the perturbative classical $n$-point block (2.3) and the on-shell worldline action of the dual network (4.15) be related to each other as

$$
f(z)=S(w)+i \sum_{k=1}^{n-2} \epsilon_{k} w_{k} .
$$

Here, the last term results from conformal transformations (5.1) of $n-2$ perturbative heavy operators. Indeed, the corresponding correlation function gains the factors $(d w / d z)^{-\epsilon_{i}}$ in the insertion points, while the quantum conformal block is exponentiated to give the classical conformal block (2.3).

Proposition 5.1 Given (5.1) and (5.3) the accessory and angular parameters are related as

$$
c_{k}=\epsilon_{k} \frac{1 \pm i \alpha s_{k}}{1-z_{k}}, \quad k=1, \ldots, n-2
$$

with the convention that "-" at $k=1$ and "+" at $k \neq 1$.

To prove the proposition we recall that both accessory parameters of perturbative heavy operators and angular parameters of the corresponding external worldlines are uniformly defined as the gradients,

$$
c_{k}=\frac{\partial f}{\partial z_{k}}, \quad s_{k}=\frac{1}{\alpha \epsilon_{k}} \frac{\partial S}{\partial w_{k}}, \quad k=1, \ldots, n-2,
$$

cf. (2.5) and (4.18). Then, the relation (5.4) directly follows from the conformal transformation (5.1) applied to the systems (5.5) supplemented with the change (5.3).

To complete the proof of the correspondence we show that the algebraic equation systems imposed on the accessory and angular parameters have the same roots. To this end, we find the same structures on both sides and partially solve the bulk system to show that the resulting equations coincide with the boundary equations.

\subsection{Weak equivalence}

On the formal level, the problem is as follows. We consider a potential vector field

$$
A_{i}(x)=\frac{\partial U(x)}{\partial x^{i}}, \quad i=1, \ldots, n-2,
$$

on a manifold with $n-2$ local coordinates $x^{i}$ and potential $U(x)$. Let the vector components $A_{i}$ be subjected to algebraic equations given implicitly by

$$
C_{\alpha}^{(N)}(A, B)=0, \quad \alpha=1, \ldots, N,
$$


where $B_{k}$ are possible auxiliary variables, $k=1, \ldots, N-(n-2)$. The coefficients in (5.7) may explicitly depend on coordinates $x$ and some additional parameters. We assume that the algebraic system (5.7) is non-degenerate and therefore the auxiliary variables can be completely expressed in terms of the potential vector field components, $B=B(A)$.

We consider two potential vector field systems defined by two different sets

$$
\begin{aligned}
& \left\{x, U(x), A(x), B(x), C^{(N)}\right\}, \\
& \left\{y, \widetilde{U}(y), \widetilde{A}(y), \widetilde{B}(y), \widetilde{C}^{(\widetilde{N})}\right\} .
\end{aligned}
$$

Definition 5.2 Two systems (5.8), (5.9) are called weakly equivalent if the implicit relations $(\alpha=1, \ldots, N$ and $\tilde{\beta}=1, \ldots, \tilde{N})$

$$
C_{\alpha}(A, B)=0, \quad C_{\tilde{\beta}}(\widetilde{A}, \widetilde{B})=0,
$$

have at least one common root $\left\{A_{i}^{0}(x)\right\} \rightarrow\left\{\widetilde{A}_{i}^{0}(y)\right\}$ under transformations

$$
x \rightarrow y, \quad U(x) \rightarrow \widetilde{U}(y) .
$$

In our case, the boundary system has no B-type variables which are characteristic of the bulk system. This is quite natural from the AdS/CFT perspective in the sense that not all bulk degrees of freedom are fundamental. Integrating out the local degrees of freedom identified here with B-type variables we are left with A-type variables which are fundamental boundary variables.

\subsection{Accessory equations}

Fixing $\epsilon_{n-1}=\epsilon_{n}$ we summarize the accessory equations (2.7) and (3.16),

$$
\begin{array}{r}
c_{1}=-\sum_{i=2}^{n-2}\left[c_{i}\left(1-z_{i}\right)-\epsilon_{i}\right]+\epsilon_{1}, \\
\left(I_{++}^{(k)}\right)^{2}+I_{-+}^{(k)} I_{+-}^{(k)}=4 \pi^{2} \tilde{\epsilon}_{k}^{2}, \quad k=1, \ldots, n-3,
\end{array}
$$

where independent variables are $c_{1}, \ldots, c_{n-2}$, while $I_{ \pm \pm}^{(k)}$ are given by (3.12), (3.13). In total, there are $(n-2)$ equations for $(n-2)$ variables.

Given the conformal map (5.1) we introduce the following notation

$$
A_{p}=a_{p}\left(1+i s_{p}\right), \quad \bar{A}_{p}=\bar{a}_{p}\left(1-i s_{p}\right), \quad \text { where } \quad a_{p}=\left(1-z_{p}\right)^{\alpha} .
$$

Insertions points satisfy the unit circle constraint (5.2) so that $\bar{a}_{p}=1 / a_{p}$. Moreover, in order to simplify the accessory equations we recover the exchanged angular momenta expressed via the external ones (4.22). As a result, we rewrite the accessory equations in terms of the external and exchanged momenta. 
Proposition 5.3 Accessory equations (5.12) and (5.13) are equivalently rewritten in terms of the angular parameters as

$$
\begin{aligned}
\epsilon_{1} s_{1}-\sum_{i=2}^{n-2} \epsilon_{i} s_{i} & =0, \\
\operatorname{Re}\left[2 \pi i \bar{A}_{k+1} I_{+-}^{(k-1)}\right] & =4 \pi^{2} \tilde{\epsilon}_{k-1}\left(s_{k+1} \tilde{s}_{k-1}+\sigma_{k+1}\right),
\end{aligned}
$$

where $k=1, \ldots, n-3$, and

$$
I_{+-}^{(k-1)}=2 \pi i\left(\epsilon_{1} \bar{A}_{1}+\sum_{p=2}^{k} \epsilon_{p} A_{p}\right),
$$

and $\sigma_{k}$ is given by (4.25), $\tilde{s}_{k}$ is given by (4.22).

The proof is given in appendix A.3.

\subsection{Momentum equations}

Using notation (5.14) we summarize the momentum equations consisting of the equilibrium conditions (4.21), (4.24), and the angular balance condition (4.30) as

$$
\begin{array}{rr}
\epsilon_{i} s_{i}+\tilde{\epsilon}_{i-1} \tilde{s}_{i-1}-\tilde{\epsilon}_{i-2} \tilde{s}_{i-2}=0, & i=2, \ldots, n-1, \\
\tilde{\epsilon}_{k-1} \sqrt{1-\tilde{s}_{k-1}^{2} \eta_{k-1}}-\tilde{\epsilon}_{k-2} \sqrt{1-\tilde{s}_{k-2}^{2} \eta_{k-1}}-\epsilon_{k} \sqrt{1-\tilde{s}_{k}^{2} \eta_{k-1}}=0, & k=2, \ldots, n-2, \\
D_{k}^{+}=\frac{\bar{A}_{k+1}}{\bar{A}_{k}} D_{k-1}^{-}, & k=2, \ldots, n-2,
\end{array}
$$

where independent variables are angular parameters $s_{1}, \ldots, s_{n-2}, \tilde{s}_{1}, \ldots, \tilde{s}_{n-3}$, and vertex positions $\eta_{1}, \ldots, \eta_{n-3}$, while $D_{k}^{ \pm}$are given by $(4.31),(4.32)$. In total, there are $(3 n-8)$ equations for $(3 n-8)$ variables.

In the bulk there are two types of redundant variables compared to those on the boundary: exchanged angular parameters $\tilde{s}_{k}$ and vertex radial positions $\eta_{k}$. These are $B$-type variables of section 5.1. The A-type variables in this case are accessory parameters and external momenta. It follows that a straightforward way to compare two descriptions is to express $\eta_{k}$ in terms of $s_{i}$ and $\tilde{s}_{j}=\tilde{s}_{j}\left(s_{i}\right)$ by (5.18), (5.19) using (4.25) and then substitute them into the angular balance equations (5.20). However, the resulting equations depending only on $s_{i}$ turn out to be very complicated. In what follows we partially solve the momentum equations that drastically simplifies the analysis of the correspondence.

To this end we notice that functions $D_{k}^{ \pm}$are building blocks of the angular balance equation (5.20). We study their properties given that the equilibrium equations (5.18) and (5.19) are satisfied and find out that they are proportional to the contour integrals $I_{+-}^{(k)}$. Two lemmas are in order. 
Lemma 5.4 Given the equilibrium conditions (5.18) and (5.19), the functions $D_{k}^{-}$(4.31) and $D_{k}^{+}$(4.32) are linearly dependent

$$
\tilde{\epsilon}_{k-1} D_{k}^{-}-\tilde{\epsilon}_{k-2} D_{k}^{+}=\epsilon_{k} A_{k} \bar{A}_{k}, \quad k=2, \ldots, n-2 .
$$

The proof is given in appendix A.4.

Lemma 5.5 Given the equilibrium conditions (5.18) and (5.19), the real part of $D_{k}^{+}$can be chosen in the form

$$
\operatorname{Re} D_{k}^{+}=-\left(s_{k} \tilde{s}_{k-2}+\sigma_{k}\right), \quad k=2, \ldots, n-2,
$$

where $\sigma_{k}$ is given by (4.25).

The proof is given in appendix A.5.

The following proposition states that the momentum equations can be reformulated in terms of new variables $D_{k}^{ \pm}$.

Proposition 5.6 Equations (5.18)-(5.20) can be rewritten as

$$
\begin{aligned}
\epsilon_{i} s_{i}+\tilde{\epsilon}_{i-1} \tilde{s}_{i-1}-\tilde{\epsilon}_{i-2} \tilde{s}_{i-2} & =0, & i & =2, \ldots, n-1, \\
\operatorname{Re} D_{k+1}^{+} & =-\left(s_{k+1} \tilde{s}_{k-1}+\sigma_{k+1}\right), & k & =1, \ldots, n-2,
\end{aligned}
$$

where $D_{k}^{-}$and $D_{k+1}^{+}, k=1, \ldots, n-2$ satisfy conditions

$$
\begin{aligned}
\tilde{\epsilon}_{k-1} D_{k}^{-}-\tilde{\epsilon}_{k-2} D_{k}^{+} & =\epsilon_{k} A_{k} \bar{A}_{k}, & k & =1, \ldots, \\
D_{k}^{+} & =\frac{\bar{A}_{k+1}}{\bar{A}_{k}} D_{k-1}^{-}, & k & =2, \ldots, n-2 .
\end{aligned}
$$

The proof directly follows from lemmas 5.4 and 5.5. Here, the vertex radial positions have been eliminated by means of introducing new independent variables $D_{k}^{ \pm}$satisfying relations (5.25) and (5.26). In this form the momentum equations are similar to the accessory equations of proposition 5.3.

\subsection{Comparing two systems}

Putting propositions 5.3 and 5.6 together we find out that the two equation systems can be weakly equivalent if the contour integrals $I_{-+}^{(k-1)}$ and functions $D_{k}^{+}$are related as $\bar{A}_{k+1} I_{-+}^{(k-1)} \sim D_{k}^{+}$. On the other hand, we might conclude that this cannot be the case because given the linear relation (5.4) the contour integrals are quadratic functions of accessory parameters, while $D_{k}^{ \pm}$are composite functions with radicals and rational functions of momenta, cf. (4.31), (4.32). However, from proposition 5.6 it follows that $D_{k}^{ \pm}$are now independent variables subjected to linear equations (5.25) and (5.26) with coefficients at most quadratic in $A_{k}$ and $\bar{A}_{k}$. Below we explicitly solve these linear relations.

First, in section 4.2 we showed that the relation (5.23) can be solved in terms of external parameters to give (5.15), see (4.21)-(4.23). 
Second, recalling that the lowest indices can be identified as $\tilde{0}=1$ implying $\tilde{\epsilon}_{0} \equiv \epsilon_{1}$ we find that equations (5.25) are explicitly written as

$$
\epsilon_{1} D_{1}^{-}=\epsilon_{1} A_{1} \bar{A}_{1}, \quad \tilde{\epsilon}_{1} D_{2}^{-}=\epsilon_{1} D_{2}^{+}+\epsilon_{2} A_{2} \bar{A}_{2}, \quad \tilde{\epsilon}_{2} D_{3}^{-}=\epsilon_{2} D_{3}^{+}+\epsilon_{3} A_{3} \bar{A}_{3}, \quad \cdots
$$

while equations (5.26) are

$$
D_{2}^{+}=\frac{\bar{A}_{2}}{\bar{A}_{1}} D_{1}^{-}, \quad D_{3}^{+}=\frac{\bar{A}_{3}}{\bar{A}_{2}} D_{2}^{-}, \quad D_{4}^{+}=\frac{\bar{A}_{4}}{\bar{A}_{3}} D_{3}^{-}, \quad \cdots .
$$

Solving (5.27) and (5.28) recursively we write down the general solution

$$
D_{k+1}^{+}=\frac{1}{\tilde{\epsilon}_{k-1}} \bar{A}_{k+1}\left(\epsilon_{1} \bar{A}_{1}+\sum_{p=2}^{k} \epsilon_{p} A_{p}\right), \quad D_{k}^{-}=\frac{1}{\tilde{\epsilon}_{k-1}} \bar{A}_{k}\left(\epsilon_{1} \bar{A}_{1}+\sum_{p=2}^{k} \epsilon_{p} A_{p}\right)
$$

Now, recalling (5.17) we find the relation

$$
D_{k+1}^{+}=\frac{1}{2 \pi i \tilde{\epsilon}_{k-1}} \bar{A}_{k+1} I_{+-}^{(k-1)}
$$

which finally proves

Proposition 5.7 The accessory equations (5.12) and (5.13) are weakly equivalent to the momentum equations (5.23) and (5.24).

The weak equivalence assumes that the bulk system has more roots compared to the boundary system. Our choice of roots is hidden in lemma 5.5. In appendix A.5 we show that there is the other possible value of $\operatorname{Re} D_{k}^{+}$. However, the corresponding root (A.25) does not give rise to known dual counterparts. In other words, it seems that there are admissible bulk configurations which cannot be realized through the classical conformal block.

\section{Conclusion}

In this work we showed that $n$-point classical conformal blocks in the heavy-light approximation are equal (modulo the conformal map) to the lengths of dual geodesic networks for any $n$. To this end we reformulated both bulk/boundary systems as the potential vector field equations, where vector components are subjected to the algebraic equations. Given the conformal map from the complex plane to cylinder we demonstrated that both algebraic systems share the same roots. This guarantees the correspondence even though explicit block and dual length functions are not presently known (except for various approximations). Moreover, using the notion of the weak equivalence we showed that the roots of the boundary system is the subset in the roots of the bulk system. The role of extra roots in the bulk will be studied elsewhere.

A possible future direction is to apply our technique to a semiclassical CFT on higher genius Riemann surfaces starting with the torus. The classical toroidal blocks were considered, e.g., in [32, 33], while their holographic interpretation in the heavy-light semiclassical 
approximation was proposed in [34]. However, the perturbative monodromy approach for toroidal and higher genius CFTs and its holographic interpretation similar to that in the spherical case have not yet been elaborated.

Also, the semiclassical correspondence considered in this paper can be extended by including $1 / c$ corrections. The 4 -point case was studied in [35-39]. It would be interesting to understand how our results for $n$-point blocks connect with going beyond the leading $1 / c$ order.

\section{Acknowledgments}

I am grateful to Vladimir Belavin for useful exchanges. I thank Bengt Nilsson and Aritra Banerjee for a stimulating discussion. Also, I would like to thank the Munich Institute for Astro- and Particle Physics (MIAPP) for hospitality during the programme "Higher-Spin Theory and Duality" (May 2016). The work was supported by RFBR grant No. 14-02-01171.

\section{A Technical details}

\section{A.1 Worldlines in the angle deficit geometry}

Here we collect main formulas of the worldline formulation on the angle deficit space $[9,12]$. The worldline action associated to the metric (4.1) is given by

$$
S=\epsilon \int_{\lambda^{\prime}}^{\lambda^{\prime \prime}} d \lambda \sqrt{\alpha^{2} \sec ^{2} \rho \dot{t}^{2}+\alpha^{2} \tan ^{2} \rho \dot{\phi}^{2}+\sec ^{2} \rho \dot{\rho}^{2}} .
$$

It is reparameterization invariant and therefore the evolution parameter can be conveniently chosen such that the proper velocity is unit. It follows that the Lagrangian function (A.1) is unit $\alpha^{2} \sec ^{2} \rho \dot{t}^{2}+\alpha^{2} \tan ^{2} \rho \dot{\phi}^{2}+\sec ^{2} \rho \dot{\rho}^{2}=1$ and the action is simply the geodesic length of a segment stretched between endpoints $\lambda^{\prime}$ and $\lambda^{\prime \prime}$. Choosing the constant time slice $t=0$ we find that the normalization condition is cast into the form

$$
\frac{p_{\phi}^{2}}{\alpha^{2}} \cot ^{2} \rho+\sec ^{2} \rho \dot{\rho}^{2}=1 .
$$

where $p_{\phi}$ is the conserved angular momentum associated with the cyclic coordinate $\phi$. A tricky point here is that we can avoid solving the geodesic equations of motion explicitly because the normalization condition (A.2) is sufficient to express a proper parameter $\lambda$ as a function of radius and angular momentum. From (A.2) we find the radial velocity

$$
\dot{\rho}= \pm \cos \rho \sqrt{1-\frac{p_{\phi}^{2}}{\alpha^{2}} \cot ^{2} \rho} .
$$

Recalling $\dot{\rho}=d \rho / d \lambda$ we find that equation (A.3) can be directly integrated to obtain the on-shell value of $S$ on the hyperbolic disk

$$
S=\left.\ln \frac{\sqrt{\eta}}{\sqrt{1+\eta}+\sqrt{1-s^{2} \eta}}\right|_{\eta^{\prime}} ^{\eta^{\prime \prime}}
$$


where $\eta^{\prime}=\cot ^{2} \rho^{\prime}$ and $\eta^{\prime \prime}=\cot ^{2} \rho^{\prime \prime}$ are initial/final radial positions. Here we used parameter $s=\left|p_{\phi}\right| / \alpha$ which is an integration constant describing the shape of a geodesic segment, cf. (4.5).

Using $p_{\phi}=g_{\phi \phi} \dot{\phi}$ and recalling that the angular momenta are integration constants we find that the angle increment of the geodesic segment is given by

$$
\Delta \phi= \pm \frac{p_{\phi}}{\alpha^{2}} \int_{\rho^{\prime}}^{\rho^{\prime \prime}} \frac{d \rho \cos \rho}{\sin ^{2} \rho\left(1-\frac{p_{\phi}^{2}}{\alpha^{2}} \cot ^{2} \rho\right)^{1 / 2}}
$$

Taking the integral we arrive at the logarithm representation (4.29).

\section{A.2 Proof of proposition 4.1}

To prove the proposition we introduce the classical fusion polynomial [12]

$$
\Pi_{I J K}=\left(\epsilon_{I}+\epsilon_{J}+\epsilon_{K}\right)\left(-\epsilon_{I}+\epsilon_{J}+\epsilon_{K}\right)\left(\epsilon_{I}-\epsilon_{J}+\epsilon_{K}\right)\left(\epsilon_{I}+\epsilon_{J}-\epsilon_{K}\right) .
$$

The polynomial has two main properties ${ }^{8}$

$$
\Pi_{I J K}=\Pi_{I K J}=\Pi_{J K I},
$$

and

$$
\Pi_{I J K}=4 \epsilon_{I}^{2} \epsilon_{J}^{2}\left(1-\sigma_{I J}^{2}\right), \quad \Pi_{I K J}=4 \epsilon_{I}^{2} \epsilon_{K}^{2}\left(1-\sigma_{I K}^{2}\right), \quad \Pi_{J K I}=4 \epsilon_{J}^{2} \epsilon_{K}^{2}\left(1-\sigma_{J K}^{2}\right),
$$

where the sigmas are given by (4.8)-(4.10). We introduce

$$
\begin{gathered}
s_{I J}=s_{I}^{2}+s_{J}^{2}-2 \sigma_{I J} s_{I} s_{J}, \\
s_{I K}=s_{I}^{2}+s_{K}^{2}-2 \sigma_{I K} s_{I} s_{K}, \\
s_{J K}=s_{J}^{2}+s_{K}^{2}+2 \sigma_{J K} s_{J} s_{K} .
\end{gathered}
$$

The vertex positions $(4.8),(4.9),(4.10)$ are then given by

$$
\eta=\left(\frac{1}{2 \epsilon_{I} \epsilon_{J}}\right)^{2} \frac{\Pi_{I J K}}{s_{I J}}, \quad \eta=\left(\frac{1}{2 \epsilon_{I} \epsilon_{K}}\right)^{2} \frac{\Pi_{I K J}}{s_{I K}}, \quad \eta=\left(\frac{1}{2 \epsilon_{J} \epsilon_{K}}\right)^{2} \frac{\Pi_{J K I}}{s_{J K}}
$$

Assume that $\epsilon_{I}+\epsilon_{J}<\epsilon_{K}$. Squaring this inequality and using (A.8) we find that $\sigma_{I J}<-1$, and $\Pi_{I J K}<0$. On the other hand, the angular momenta are non-negative $s_{I, J, K} \geq 0$, so that if $\sigma_{I J}<-1$ then $s_{I J} \geq 0$. Using for $\eta$ the first representation in (A.10) we conclude that $\eta<0$. It follows that $\epsilon_{I}+\epsilon_{J} \geq \epsilon_{K}$ is necessary to have real vertex positions.

The second triangle inequality is proved along the same lines. Suppose that $\epsilon_{I}+\epsilon_{K}<$ $\epsilon_{J}$. Using for $\eta$ the second representation in (A.10) we conclude that $\eta \geq 0$ provided $\epsilon_{I}+\epsilon_{K} \geq \epsilon_{J}$.

\footnotetext{
${ }^{8}$ Note that the classical fusion polynomial is just the Heron's function defining the area of the triangle Area $_{\Delta}\left(\epsilon_{I}, \epsilon_{J}, \epsilon_{K}\right)=\frac{1}{4} \sqrt{\Pi\left(\epsilon_{I}, \epsilon_{J}, \epsilon_{K}\right)}$ in the space of conformal dimensions.
} 
Now, suppose that $\epsilon_{J}+\epsilon_{K}<\epsilon_{I}$. In this case, it is convenient to represent $\eta$ by the third formula in (A.10), where $\sigma_{J K}$ in $s_{J K}$ has an opposite sign compared to $s_{I J}$ and $s_{I K}$, cf. (A.9). Substituting the other two established triangle inequalities $\epsilon_{I}+\epsilon_{J} \geq \epsilon_{K}$ and $\epsilon_{I}+\epsilon_{K} \geq \epsilon_{J}$ into $\Pi_{J K I}$ we find that $\Pi_{J K I} \geq 0$. On the other hand, squaring $\epsilon_{J}+\epsilon_{K} \geq \epsilon_{I}$ yields $\sigma_{J K}>1$, hence $s_{J K} \geq 0$.

We conclude that having the triangle inequalities (4.12) is a necessary condition for $\eta \geq 0$.

\section{A.3 Proof of proposition 5.3}

Recalling that the heavy background dimensions are equal, $\epsilon_{n-1}=\epsilon_{n}$, we find that (2.7) takes the form

$$
c_{1}-\epsilon_{1}=-\sum_{i=2}^{n-2}\left[c_{i}\left(1-z_{i}\right)-\epsilon_{i}\right] .
$$

Under the correspondence map (5.4) it transforms to the relation between the external angular parameters (4.23),

$$
\epsilon_{1} s_{1}-\sum_{i=2}^{n-2} \epsilon_{i} s_{i}=0
$$

Now, using (5.4) the contour integrals (3.12)-(3.13) can be expressed in terms of external angular parameters $s_{k}, k=1, \ldots, n-3$. Using notation (5.14) we find that the contour integrals are given by

$$
\begin{aligned}
& I_{+-}^{(k)}=2 \pi i\left[\epsilon_{1} \bar{A}_{1}+\sum_{p=2}^{k+1} \epsilon_{p} A_{p}\right] \equiv I_{+-}^{(k-1)}+2 \pi i \epsilon_{k+1} A_{k+1}, \\
& I_{-+}^{(k)}=2 \pi i\left[\epsilon_{1} A_{1}+\sum_{p=2}^{k+1} \epsilon_{p} \bar{A}_{p}\right] \equiv I_{-+}^{(k-1)}+2 \pi i \epsilon_{k+1} \bar{A}_{k+1}, \\
& I_{++}^{(k)}=2 \pi\left[-\epsilon_{1} s_{1}+\sum_{p=2}^{k+1} \epsilon_{p} s_{p}\right] \equiv I_{++}^{(k-1)}+2 \pi i \epsilon_{k+1} s_{k+1},
\end{aligned}
$$

with the convention that $I_{+-}^{(0)} \equiv 2 \pi i \epsilon_{1} \bar{A}_{1}$ and $I_{++}^{(0)} \equiv-2 \pi \epsilon_{1} s_{1}$. In particular, as a consistency check we find from (4.23) that $I_{++}^{(n-3)}=0$, see our comment below (3.13).

The accessory equations (5.12) are then represented as

$$
\left(-\epsilon_{1} s_{1}+\sum_{p=2}^{k+1} \epsilon_{p} s_{p}\right)^{2}-\left(\epsilon_{1} \bar{A}_{1}+\sum_{p=2}^{k+1} \epsilon_{p} A_{p}\right)\left(\epsilon_{1} A_{1}+\sum_{p=2}^{k+1} \epsilon_{p} \bar{A}_{p}\right)+\tilde{\epsilon}_{k}^{2}=0
$$

where $k=1,2, \ldots, n-3$. Equations (A.16) at $k$ and $k-1$ are related as follows. We substitute the right-hand side equalities of (A.13)-(A.15) into (A.16) and find that

$$
4 \pi s_{k+1} I_{++}^{(k-1)}+2 \pi i\left(A_{k+1} I_{-+}^{(k-1)}+\bar{A}_{k+1} I_{+-}^{(k-1)}\right)=8 \pi^{2} \tilde{\epsilon}_{k-1} \sigma_{k+1},
$$


where the sigma is given by (4.25). Furthermore, we note that $I_{++}^{(k-1)}$ can be expressed in terms of the exchanged angular parameters by means of the relation (4.22), namely, $I_{++}^{(k-1)}=-2 \pi \tilde{\epsilon}_{k-1} \tilde{s}_{k-1}$. Finally, we get

$$
2 \pi i\left(A_{k+1} I_{-+}^{(k-1)}+\bar{A}_{k+1} I_{+-}^{(k-1)}\right)=8 \pi^{2} \tilde{\epsilon}_{k-1}\left(\tilde{s}_{k-1} s_{k+1}+\sigma_{k+1}\right) .
$$

Noting that $\left(I_{-+}^{(k)}\right)^{*}=-I_{+-}^{(k)}$, where $*$ denotes the complex conjugation and using $\operatorname{Re} x=$ $\left(x+x^{*}\right) / 2$ we conclude that the resulting equation is exactly (5.16), while (5.17) is (A.13).

\section{A.4 Proof of lemma 5.4}

Following definition (4.31) and (4.32) we consider the difference

$$
\tilde{\epsilon}_{k-1} D_{k}^{-}-\tilde{\epsilon}_{k-2} D_{k}^{+} \equiv\left(\sqrt{1-s_{k}^{2} \eta_{k-1}}-i s_{k} \sqrt{1+\eta_{k-1}}\right) \Delta B_{k}
$$

where

$$
\begin{aligned}
\Delta B_{k}= & \tilde{\epsilon}_{k-1}\left(\sqrt{1-\tilde{s}_{k-1}^{2} \eta_{k-1}}-i \tilde{s}_{k-1} \sqrt{1+\eta_{k-1}}\right) \\
& -\tilde{\epsilon}_{k-2}\left(\sqrt{1-\tilde{s}_{k-2}^{2} \eta_{k-1}}-i \tilde{s}_{k-2} \sqrt{1+\eta_{k-1}}\right) \\
= & \left(\tilde{\epsilon}_{k-1} \sqrt{1-\tilde{s}_{k-1}^{2} \eta_{k-1}}-\tilde{\epsilon}_{k-2} \sqrt{1-\tilde{s}_{k-2}^{2} \eta_{k-1}}\right)+i\left(\tilde{\epsilon}_{k-2} \tilde{s}_{k-2}-\tilde{\epsilon}_{k-1} \tilde{s}_{k-1}\right) .
\end{aligned}
$$

The real and imaginary parts here are given by the equilibrium conditions (5.19) and (5.18), respectively. It follows that

$$
\Delta B_{k}=\epsilon_{k}\left(\sqrt{1+s_{k}^{2} \eta_{k-1}}+i \sqrt{1+\eta_{k-1}}\right)
$$

which is modulo $\epsilon_{k}$ is the complex conjugate of the first factor in (A.19). Using (5.2) and (5.14) we find that the absolute value of this number is $1+s_{k}^{2}=A_{k} \bar{A}_{k}$. Thus, (5.21) holds true.

\section{A.5 Proof of lemma 5.5}

Here we partially solve the momentum equations. To this end, we solve (4.32) in terms of the radial vertex positions

$$
\eta_{k-1}=\frac{1-\left(\operatorname{Re} D_{k}^{+}+s_{k} \tilde{s}_{k-2}\right)^{2}}{s_{k}^{2}+\tilde{s}_{k-2}^{2}+2 s_{k} \tilde{s}_{k-2}\left(\operatorname{Re} D_{k}^{+}+s_{k} \tilde{s}_{k-2}\right)},
$$

where $D_{k}^{+}$is not arbitrary but restricted by the balance equation (4.30). The real part is conveniently defined as $\operatorname{Re} D_{k}^{+}=\left(D_{k}^{+}+D_{k}^{+*}\right) / 2$, where $*$ denotes the complex conjugation. There are no other solutions since equations (4.32) are solved by squaring the radicals: squaring twice we obtain linear in $\eta_{k}$ equations (see our comments below (4.8)).

Equating two different but equivalent representations (4.25) and (A.22) of the vertex radial position $\eta_{k-1}$ we find that the real part of $D_{k}^{+}$satisfies the quadratic equation

$$
\frac{1-\sigma_{k}^{2}}{s_{k}^{2}+\tilde{s}_{k-2}^{2}-2 \sigma_{k} s_{k} \tilde{s}_{k-2}}=\frac{1-\left(\operatorname{Re} D_{k}^{+}+s_{k} \tilde{s}_{k-2}\right)^{2}}{s_{k}^{2}+\tilde{s}_{k-2}^{2}+2 s_{k} \tilde{s}_{k-2}\left(\operatorname{Re} D_{k}^{+}+s_{k} \tilde{s}_{k-2}\right)} .
$$


There are two different roots at each $k=2, \ldots, n-2$,

$$
\begin{aligned}
\operatorname{Re} D_{k}^{+}+s_{k} \tilde{s}_{k-2}+\sigma_{k} & =0, \\
\operatorname{Re} D_{k}^{+}+s_{k} \tilde{s}_{k-2}-\sigma_{k} \frac{s_{k}^{2}+\tilde{s}_{k-2}^{2}-2 \sigma_{k}^{-1} s_{k} \tilde{s}_{k-2}}{s_{k}^{2}+\tilde{s}_{k-2}^{2}-2 \sigma_{k} s_{k} \tilde{s}_{k-2}} & =0 .
\end{aligned}
$$

In what follows we choose just the first root (A.24), see our comments below proposition 5.7.

Open Access. This article is distributed under the terms of the Creative Commons Attribution License (CC-BY 4.0), which permits any use, distribution and reproduction in any medium, provided the original author(s) and source are credited.

\section{References}

[1] J.M. Maldacena, The Large- $N$ limit of superconformal field theories and supergravity, Int. J. Theor. Phys. 38 (1999) 1113 [Adv. Theor. Math. Phys. 2 (1998) 231] [hep-th/9711200] [INSPIRE].

[2] E. Witten, Anti-de Sitter space and holography, Adv. Theor. Math. Phys. 2 (1998) 253 [hep-th/9802150] [INSPIRE].

[3] S.S. Gubser, I.R. Klebanov and A.M. Polyakov, Gauge theory correlators from noncritical string theory, Phys. Lett. B 428 (1998) 105 [hep-th/9802109] [INSPIRE].

[4] T. Hartman, Entanglement Entropy at Large Central Charge, arXiv:1303.6955 [INSPIRE].

[5] A.L. Fitzpatrick, J. Kaplan and M.T. Walters, Universality of Long-Distance AdS Physics from the CFT Bootstrap, JHEP 08 (2014) 145 [arXiv: 1403.6829] [INSPIRE].

[6] C.T. Asplund, A. Bernamonti, F. Galli and T. Hartman, Holographic Entanglement Entropy from 2d CFT: Heavy States and Local Quenches, JHEP 02 (2015) 171 [arXiv:1410.1392] [INSPIRE].

[7] P. Caputa, J. Simón, A. Štikonas and T. Takayanagi, Quantum Entanglement of Localized Excited States at Finite Temperature, JHEP 01 (2015) 102 [arXiv:1410.2287] [INSPIRE].

[8] J. de Boer, A. Castro, E. Hijano, J.I. Jottar and P. Kraus, Higher spin entanglement and $\mathcal{W}_{\mathrm{N}}$ conformal blocks, JHEP 07 (2015) 168 [arXiv:1412.7520] [INSPIRE].

[9] E. Hijano, P. Kraus and R. Snively, Worldline approach to semi-classical conformal blocks, JHEP 07 (2015) 131 [arXiv:1501.02260] [INSPIRE].

[10] A.L. Fitzpatrick, J. Kaplan and M.T. Walters, Virasoro Conformal Blocks and Thermality from Classical Background Fields, JHEP 11 (2015) 200 [arXiv:1501.05315] [INSPIRE].

[11] E. Perlmutter, Virasoro conformal blocks in closed form, JHEP 08 (2015) 088 [arXiv: 1502.07742] [INSPIRE].

[12] K.B. Alkalaev and V.A. Belavin, Classical conformal blocks via AdS/CFT correspondence, JHEP 08 (2015) 049 [arXiv: 1504.05943] [INSPIRE].

[13] E. Hijano, P. Kraus, E. Perlmutter and R. Snively, Semiclassical Virasoro blocks from $A d S_{3}$ gravity, JHEP 12 (2015) 077 [arXiv:1508.04987] [INSPIRE].

[14] K.B. Alkalaev and V.A. Belavin, Monodromic vs geodesic computation of Virasoro classical conformal blocks, Nucl. Phys. B 904 (2016) 367 [arXiv:1510.06685] [INSPIRE]. 
[15] P. Banerjee, S. Datta and R. Sinha, Higher-point conformal blocks and entanglement entropy in heavy states, JHEP 05 (2016) 127 [arXiv:1601.06794] [INSPIRE].

[16] B. Chen, J.-q. Wu and J.-j. Zhang, Holographic Description of $2 D$ Conformal Block in Semi-classical Limit, JHEP 10 (2016) 110 [arXiv:1609.00801] [INSPIRE].

[17] A.B. Zamolodchikov, Conformal Symmetry in Two-dimensional Space: Recursion Representation of the Conformal Block, Theor. Math. Phys. 73 (1987) 1088 [Teor. Mat. Fiz. 73 (1987) 103].

[18] V. Balasubramanian and S.F. Ross, Holographic particle detection, Phys. Rev. D 61 (2000) 044007 [hep-th/9906226] [InSPIRE].

[19] J. Louko, D. Marolf and S.F. Ross, On geodesic propagators and black hole holography, Phys. Rev. D 62 (2000) 044041 [hep-th/0002111] [INSPIRE].

[20] I.Y. Aref'eva and M.A. Khramtsov, AdS/CFT prescription for angle-deficit space and winding geodesics, JHEP 04 (2016) 121 [arXiv: 1601.02008] [INSPIRE].

[21] A.A. Belavin, A.M. Polyakov and A.B. Zamolodchikov, Infinite Conformal Symmetry in Two-Dimensional Quantum Field Theory, Nucl. Phys. B 241 (1984) 333 [inSPIRE].

[22] A. Litvinov, S. Lukyanov, N. Nekrasov and A.B. Zamolodchikov, Classical Conformal Blocks and Painleve VI, JHEP 07 (2014) 144 [arXiv:1309.4700] [InSPIRE].

[23] A.-K. Kashani-Poor and J. Troost, Transformations of Spherical Blocks, JHEP 10 (2013) 009 [arXiv: 1305.7408] [INSPIRE].

[24] K.B. Alkalaev and V.A. Belavin, From global to heavy-light: 5-point conformal blocks, JHEP 03 (2016) 184 [arXiv: 1512.07627] [INSPIRE].

[25] L. Cantini, P. Menotti and D. Seminara, Liouville theory, accessory parameters and (2+1)-dimensional gravity, Nucl. Phys. B 638 (2002) 351 [hep-th/0203103] [INSPIRE].

[26] L. Hadasz, Z. Jaskolski and M. Piatek, Classical geometry from the quantum Liouville theory, Nucl. Phys. B 724 (2005) 529 [hep-th/0504204] [INSPIRE].

[27] M. Nishida and K. Tamaoka, Geodesic Witten diagrams with an external spinning field, arXiv: 1609.04563 [INSPIRE].

[28] D. Harlow, J. Maltz and E. Witten, Analytic Continuation of Liouville Theory, JHEP 12 (2011) 071 [arXiv:1108.4417] [INSPIRE].

[29] N. Seiberg, Notes on quantum Liouville theory and quantum gravity, Prog. Theor. Phys. Suppl. 102 (1990) 319 [INSPIRE].

[30] M. Bañados, Three-dimensional quantum geometry and black holes, AIP Conf. Proc. 484 (1999) 147 [hep-th/9901148] [INSPIRE].

[31] A.B. Zamolodchikov and A.B. Zamolodchikov, Lectures on Liouville Theory and Matrix Models, (2007) and online pdf version at http://qft.itp.ac.ru/ZZ.pdf.

[32] P. Menotti, Riemann-Hilbert treatment of Liouville theory on the torus, J. Phys. A 44 (2011) 115403 [arXiv: 1010.4946] [inSPIRE].

[33] M. Piatek, Classical torus conformal block, $\mathcal{N}=2^{*}$ twisted superpotential and the accessory parameter of Lamé equation, JHEP 03 (2014) 124 [arXiv: 1309.7672] [INSPIRE].

[34] K.B. Alkalaev and V.A. Belavin, Holographic interpretation of 1-point toroidal block in the semiclassical limit, JHEP 06 (2016) 183 [arXiv:1603.08440] [INSPIRE]. 
[35] M. Beccaria, A. Fachechi and G. Macorini, Virasoro vacuum block at next-to-leading order in the heavy-light limit, JHEP 02 (2016) 072 [arXiv:1511.05452] [INSPIRE].

[36] A.L. Fitzpatrick and J. Kaplan, Conformal Blocks Beyond the Semi-Classical Limit, JHEP 05 (2016) 075 [arXiv:1512.03052] [INSPIRE].

[37] A.L. Fitzpatrick, J. Kaplan, D. Li and J. Wang, On information loss in $A d S_{3} / C F T_{2}$, JHEP 05 (2016) 109 [arXiv: 1603.08925] [INSPIRE].

[38] H. Chen, A.L. Fitzpatrick, J. Kaplan, D. Li and J. Wang, Degenerate Operators and the 1/c Expansion: Lorentzian Resummations, High Order Computations and Super-Virasoro Blocks, arXiv: 1606.02659 [INSPIRE].

[39] A.L. Fitzpatrick and J. Kaplan, On the Late-Time Behavior of Virasoro Blocks and a Classification of Semiclassical Saddles, arXiv:1609.07153 [INSPIRE]. 